\title{
Arterial Stiffness and Dialysis Calcium Concentration
}

\author{
Fabrice Mac-Way, Amélie Leboeuf, and Mohsen Agharazii \\ Division of Nephrology and Research Center of CHUQ, L'Hôtel Dieu de Québec Hospital and Faculty of Medicine, \\ Laval University, Quebec, QC, Canada G1R 2J6 \\ Correspondence should be addressed to Mohsen Agharazii, mohsen.agharazii@crhdq.ulaval.ca
}

Received 31 October 2010; Accepted 2 March 2011

Academic Editor: Biagio Raffaele Di Iorio

Copyright ( 2011 Fabrice Mac-Way et al. This is an open access article distributed under the Creative Commons Attribution License, which permits unrestricted use, distribution, and reproduction in any medium, provided the original work is properly cited.

\begin{abstract}
Arterial stiffness is the major determinant of isolated systolic hypertension and increased pulse pressure. Aortic stiffness is also associated with increased cardiovascular morbidity and mortality in patients with chronic kidney disease, hypertension, and general population. Hemodynamically, arterial stiffness results in earlier aortic pulse wave reflection leading to increased cardiac workload and decreased myocardial perfusion. Although the clinical consequence of aortic stiffness has been clearly established, its pathophysiology in various clinical conditions still remains poorly understood. The aim of the present paper is to review the studies that have looked at the impact of dialysis calcium concentration on arterial stiffness. Overall, the results of small short-term studies suggest that higher dialysis calcium is associated with a transient but significant increase in arterial stiffness. This calcium dependant increase in arterial stiffness is potentially explained by increased vascular smooth muscle tone of the conduit arteries and is not solely explained by changes in mean blood pressure. However, the optimal DCa remains to be determined, and long term studies are required to evaluate its impact on the progression of arterial stiffness.
\end{abstract}

\section{Introduction}

Midsize and large-size arteries are primarily responsible for carrying blood from the heart to the tissues (conductive function) and contribute little to the total vascular resistance. However, the arterial tree must also cope with the cyclic cardiac output and assure the perfusion of organs even during diastole. In this respect, aorta, with its unique elastic capacity, plays a vital role in dampening of the peak systolic pressure and uses its elastic recoil to assure blood flow to the organs even during diastole. Consequently, aortic stiffness, a major determinant of isolated systolic hypertension and increased pulse pressure, has been associated with increased cardiovascular events and mortality [1-5]. The mechanisms of arterial stiffness in various clinical conditions still remain poorly understood. It is thought that stiffness of central elastic arteries is the result of fragmentation of elastin fibers, increased extracellular matrix production of collagen (vascular fibrosis), modification of extracellular matrix by advances glycation end-products, and medial vascular calcification (Mönckeberg sclerosis) [6-10].

\section{Arterial Stiffness and Its Hemodynamic Consequences}

Arterial stiffness is best evaluated by determination of pulse wave velocity (PWV) over the arterial segment of interest (Figure 1). Determination of PWV as a measure of arterial stiffness provides an intuitive understanding of its impact on central (aortic) hemodynamic consequences (Figure 2). Accordingly, in patients with normal arteries, the pressure wave that is generated from the heart travels at a lower speed, hits the reflecting sites, and returns to the ascending aorta during the diastole. This late wave reflection results in the elevation of diastolic pressure and is beneficial for the coronary perfusion. However, when the blood vessels become stiff, the pressure wave travels much faster, hits the reflecting sites, and returns to the ascending aorta during the left ventricular ejection time (systole), when the aortic valves are still open. This early reflection that occurs during the systole imposes an increased workload for the left ventricle, and its absence during the diastole contributes to a rapid decay in diastolic pressure and poorer coronary perfusion pressure (Figures 3 and 4 ). 


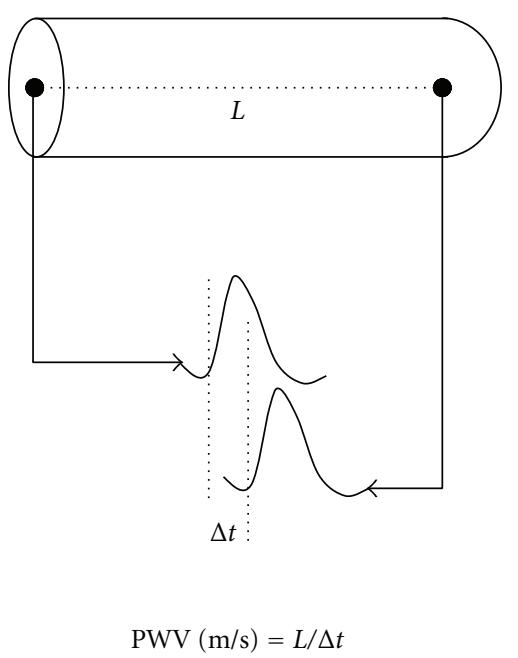

Figure 1: Pulse wave velocity. Pulse wave velocity (PWV) is measured by dividing the length of the arterial segment by the transit time of the pulse wave between the two sites of interest.

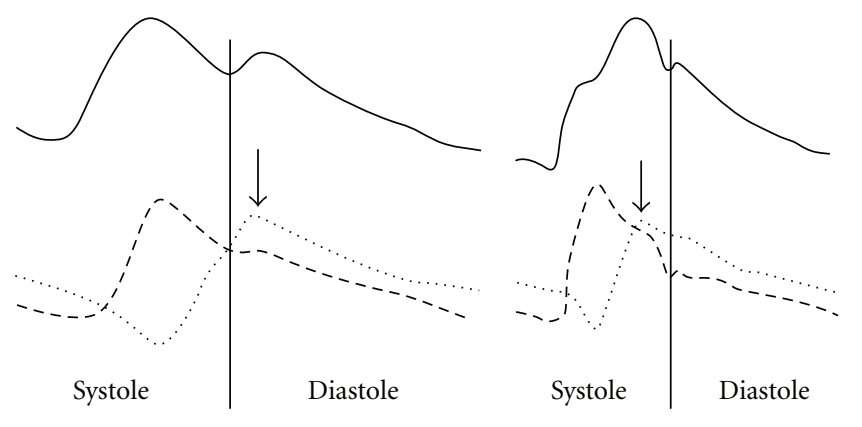

(a)

(b)

FIGURE 2: Arterial wave reflection. The upper section of both panels shows the recorded wave in subject with lower arterial stiffness (a) and higher arterial stiffness (b) as determined by a lower and a higher pulse wave respectively. The lower section of both panels shows the dissection of the recorded wave into the incidental (- - -) and reflected waves (...) in the respective conditions. In (a), it can be seen that the timing of peak reflected pressure wave (arrow) occurs after the closer of the valves (vertical line). In (b), the timing of peak reflected pressure wave (arrow) occurs before the closer of aortic valves (vertical line).

The biomechanical property of conduit arteries is heterogeneous in the arterial tree. As a general rule, the blood vessels which are closer to the heart are more elastic, and they become stiffer as they move towards the periphery. This is mostly explained by the elastin component of the vessel which follows the same pattern of distribution $[6,11]$. Although arterial stiffness can affect any conduit artery, vascular stiffness hampers mostly the unique and vital function of elastic vessels such as aorta and common carotid arteries. It is therefore not surprising that aortic stiffness has been associated with increased mortality whereas stiffness of muscular conduit arteries fails to provide this distinctive prognostic information [12]. In a recent meta-analysis of 17

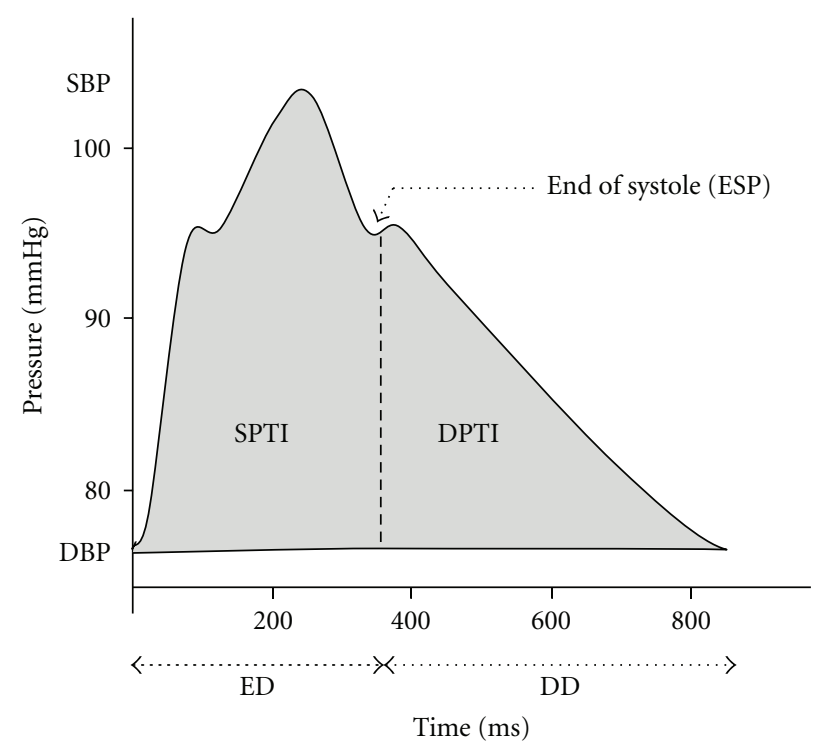

FIgure 3: Central systolic pressure time index (SPTI) and diastolic pressure time index (DPTI). Pulse wave profile analysis of ascending aorta shows that systolic pressure time index (SPTI) represents myocardial workload and the diastolic pressure time index (DPTI) represents the myocardial perfusion. The ratio of DPTI/SPTI is also referred to as the subendocardial viability ratio. Arterial stiffness and earlier wave reflection lead to a lower DPTI/SPTI ratio that may be detrimental to the myocardial function. The pulse wave profile shows also the ejection duration (ED), diastolic duration (DD), and end systolic pressure (ESP).

longitudinal studies that evaluated aortic PWV and followed up 15,877 subjects for a mean of 7.7 years, Vlachopoulos and colleagues have concluded that an increase in aortic PWV by $1 \mathrm{~m} / \mathrm{s}$ corresponded to an age-, sex-, and risk factor-adjusted risk increase of 14,15 , and $15 \%$ in total cardiovascular events, mortality, and all-cause mortality, respectively [5].

Besides the increased cardiac workload and reduced coronary perfusion, stiffness of the central elastic arteries can also affect other organs such as kidneys and brain. When these central arteries are provided with great elasticity, they can absorb the pulsatility of the flow to highly perfused organs (i.e., brain and kidneys). However, when elastic arteries become stiff, they lose the capacity to dampen this pulsatility of blood flow, and therefore, perfusion of brain and kidneys become highly pulsatile. This may explain the relationship between aortic stiffness and increased risk of stroke and microalbuminuria [13-16].

\section{Determination of Arterial Stiffness}

A thorough review of evaluation of arterial stiffness is beyond the scope of this paper and has recently been reviewed in detail by Adji and colleagues [17]. A brief presentation on determination of arterial stiffness is however necessary for the understanding of the role of dialysis calcium concentration (DCa) on arterial stiffness. It should be first mentioned that arterial stiffness assessment can be performed for local, segmental, or systemic arterial tree. The terms arterial 


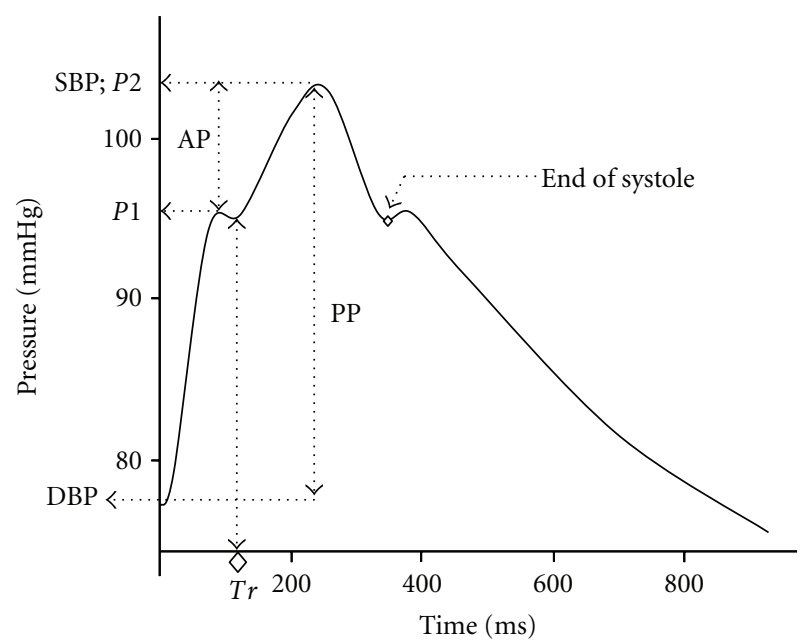

Figure 4: Central pulse wave profile. The central pulse wave profile can be broken into the following parameters: diastolic blood pressure (DBP), first peak of pressure $(P 1)$, time of return of the reflected wave $(\mathrm{Tr})$, second peak of pressure $(P 2)$, systolic blood pressure (SBP), and pulse pressure (PP). Augmented pressure (AP), reflecting the amount of central pressure increase that is due to the earlier arrival of reflected wave, can be calculated by subtracting $P 2$ from $P 1$.

stiffness, elasticity, distention, and compliance are sometimes used interchangeably in common language. However, these parameters have distinct mechanical definitions and only underline the complexity of the biomechanical property of the arterial wall. These definitions and methodological aspects of evaluation of arterial stiffness have been addressed by Laurent et al. [18] in an expert consensus document on arterial stiffness applications.

As mentioned previously, arterial stiffness is still best measured by determination of segmental PWV over that arterial segment. As arterial stiffness affects pulse wave profile, peripheral pulse wave profile analysis by radial artery tonometry, digital volume pulse, and oscillometric waveform analysis of brachial artery have been used to derive various indices of arterial stiffness and arterial compliance. For the purpose of this paper a detailed analysis of each method used to evaluate the impact of DCa on arterial stiffness will be addressed for each study.

\section{Impact of Dialysis Calcium Concentration on Arterial Stiffness in Hemodialysis}

The hemodynamic effects of higher DCa have been the subject of many studies. From these studies, one can conclude that increased cardiac output and increased vascular resistance are responsible for a lesser decrease in postdialysis blood pressure and for a hemodynamically more stable hemodialysis session [19-22]. However, only a handful of protocols have studied the impact of DCa on arterial stiffness. These studies are summarized in Table 1 and will be reviewed here in detail. In reviewing these studies, one must also consider the gradual trend towards a lower DCa that has occurred during the past two decades.

The first of these studies was performed by Marchais and colleagues [23], where they showed in hemodialysis patients $(n=26)$ that DCa of $1.5 \mathrm{mM}$ was not associated with changes in aortic and brachial stiffness as measured by PWV, whereas a DCa of $1.75 \mathrm{mM}$ was associated with an increase in both aortic $(1091 \pm 329$ to $1221 \pm 268 \mathrm{~cm} / \mathrm{s}, P<.01)$ and brachial PWV $(1173 \pm 315$ to $1438 \pm 271 \mathrm{~cm} / \mathrm{s}, P<$ $.001)$. The increase in serum ionized calcium (iCa) was more pronounced with DCa of $1.75 \mathrm{mM}(1.29 \pm 0.06$ to $1.55 \pm$ $0.01 \mathrm{mM}, P<.0001)$ than with DCa of $1.5 \mathrm{mM}(1.28 \pm 0.08$ to $1.34 \pm 0.11, P<.01)$. The blood pressure did not change during dialysis, and it was concluded that high-calcium bath induced hypercalcemia and elevation of arterial stiffness in hemodialysis patients.

In a study by Kyriazis et al. [24], the interdialytic and intradialytic effects of DCa were evaluated in 19 subjects who were randomly assigned to DCa of 1.25 and $1.75 \mathrm{mM}$ for 4 sessions of dialysis in a crossover fashion. They evaluated arterial stiffness by oscillometric waveform analysis of the brachial artery to determine brachial artery compliance, that is, the capacity of the artery to accept a volume of blood for each $\mathrm{mmHg}$ increase of pressure. The vascular compliance was derived by radial (perpendicular to the wall of the artery) compression and expansion of the brachial artery caused by the oscillating blood pressure. Determination of arterial compliance was then calculated based on the brachial artery diameter that was estimated by a mathematical model in which the average size of the brachial artery at mean arterial pressure was scaled for body surface area. Using this method, these investigators showed that predialysis pulse pressure and iCa levels were the only determinants of the predialysis brachial arterial compliance. After 4 sessions of dialysis with the lower concentration of calcium, the predialysis iCa was lower $(1.10 \pm 0.08$ versus $1.15 \pm 0.07 \mathrm{mM}, P<.001)$ and arterial compliance was better as compared to the higher DCa $(0.101 \pm 0.03$ versus $0.092 \pm 0.02 \mathrm{~mL} / \mathrm{mmHg}, P<$ $.05)$. However, the postdialysis brachial artery compliance improved by $32 \%$ and $37 \%$ with both DCa of 1.25 and 1.75 $\mathrm{mM}$, respectively. This intradialytic improvement of arterial compliance was inversely correlated with changes in systolic and pulse pressures and was not related to the changes in iCa levels. However, this lack of difference in intradialytic changes of arterial compliance between the two DCa may be related to the methodological limitations in the assessment of arterial compliance.

In 8 patients with a baseline dialysis calcium of $1.75 \mathrm{mM}$, Yoo and colleagues [25] studied the arterial compliance of the common carotid artery after ten sessions of DCa of $1.25 \mathrm{mM}$ followed by 10 sessions of DCa of $1.75 \mathrm{mM}$. The common carotid diameter was determined by ultrasound; however, the local pulse pressure was not determined, and the brachial pulse pressure was used to determine carotid compliance. Nevertheless, these investigators showed that switching to DCa of $1.25 \mathrm{mM}$ for ten sessions improved the compliance of common carotid artery which increased from $0.140 \mathrm{~mm}^{2} / \mathrm{kPa}$ to $0.170 \mathrm{~mm}^{2} / \mathrm{kPa}$. After switching back to DCa of $1.75 \mathrm{mM}$, the common carotid compliance returned 
TABLE 1: Summary of studies evaluating the effects of dialysis calcium concentration on arterial stiffness in dialysis.

\begin{tabular}{|c|c|c|c|c|c|}
\hline References & $\begin{array}{l}\text { Population } \\
\qquad(n)\end{array}$ & Dialysis Ca & Duration & Stiffness index & Results \\
\hline $\begin{array}{l}\text { Marchais et al. } \\
\text { (1989) [23] }\end{array}$ & $26 \mathrm{HD}$ & $\begin{array}{l}1.5 \mathrm{mM} \text { versus } \\
1.75 \mathrm{mM}\end{array}$ & $1 \mathrm{HD}$ per $\mathrm{DCa}$ & Aortic PWV, brachial PWV & $\begin{array}{l}\text { (i) } 1.5 \mathrm{mM} \text { : Slight } \uparrow \mathrm{iCa} \text {, } \\
\text { brachial and aortic PWV } \\
\text { unchanged } \\
\text { (ii) } 1.75 \mathrm{mM}: \uparrow \mathrm{iCa}, \uparrow \\
\text { brachial and aortic PWV }\end{array}$ \\
\hline $\begin{array}{l}\text { Kyriazis et al. } \\
(2000)[24]\end{array}$ & $19 \mathrm{HD}$ & $\begin{array}{c}1.25 \mathrm{mM} \text { versus } \\
1.75 \mathrm{mM} \text { (crossover) }\end{array}$ & $4 \mathrm{HD}$ per $\mathrm{DCa}$ & $\begin{array}{l}\text { Estimated brachial artery compliance } \\
\text { (oscillometric pulse wave analysis and } \\
\text { estimation of brachial artery diameter) }\end{array}$ & $\begin{array}{l}\text { (i) } 1.25 \mathrm{mM}: \mathrm{iCa} \text { stable, } \uparrow \\
\text { AC } \\
\text { (ii) } 1.75 \mathrm{mM}: \uparrow \mathrm{iCa}, \uparrow \mathrm{AC}\end{array}$ \\
\hline $\begin{array}{l}\text { Yoo et al. } \\
(2004)[25]\end{array}$ & $8 \mathrm{HD}$ & $\begin{array}{l}1.75 \mathrm{mM} \text { (baseline) } \downarrow \\
1.25 \mathrm{mM} \downarrow 1.75 \mathrm{mM}\end{array}$ & $10 \mathrm{HD}$ per $\mathrm{DCa}$ & $\begin{array}{l}\text { Carotid arterial compliance (carotid } \\
\text { ultrasound and brachial pulse pressure) }\end{array}$ & $\begin{array}{l}\text { (i) } 1.25 \mathrm{mM}: \downarrow \mathrm{iCa}, \uparrow \mathrm{AC} \\
\text { (ii) } 1.75 \mathrm{mM}: \uparrow \mathrm{iCa}, \downarrow \mathrm{AC}\end{array}$ \\
\hline $\begin{array}{l}\text { Kyriazis et al. } \\
(2007)[26]\end{array}$ & $14 \mathrm{HD}$ & $\begin{array}{l}1.25 \mathrm{mM} \text { versus } \\
1.75 \mathrm{mM}\end{array}$ & $1 \mathrm{HD}$ per $\mathrm{DCa}$ & $\begin{array}{l}\text { Stiffness index (SI), reflection index } \\
\text { (RI) derived from the digital volume } \\
\text { pulse waveform }\end{array}$ & $\begin{array}{l}\text { (i) } 1.25 \mathrm{mM}: \downarrow \mathrm{iCa}, \mathrm{SI} \text { and } \\
\mathrm{RI} \text { unchanged } \\
\text { (ii) } 1.75 \mathrm{mM}: \uparrow \mathrm{iCa}, \uparrow \mathrm{SI} \text {, } \\
\uparrow \mathrm{RI}\end{array}$ \\
\hline $\begin{array}{l}\text { Leboeuf et al. } \\
\text { (2009) [29] }\end{array}$ & $18 \mathrm{HD}$ & $\begin{array}{l}1.00 \mathrm{mM} \text { versus } \\
1.25 \mathrm{mM} \text { versus } \\
1.50 \mathrm{mM} \text { (Latin } \\
\text { square crossover) }\end{array}$ & $1 \mathrm{HD}$ per $\mathrm{DCa}$ & $\begin{array}{l}\text { Brachial PWV, aortic PWV, } \\
\text { augmentation index (AI) }\end{array}$ & $\begin{array}{l}\text { (i) Association between } \\
\Delta \mathrm{iCa} \text { and relative changes } \\
\text { in brachial and aortic } \\
\text { stiffness, independent of BP } \\
\text { (ii) Postdialysis reduction } \\
\text { of augmentation index, } \\
\text { independent of DCa or } \\
\Delta \mathrm{iCa}\end{array}$ \\
\hline $\begin{array}{l}\text { Demirci et al. } \\
(2008) \text { [30] }\end{array}$ & $49 \mathrm{PD}$ & $\begin{array}{c}1.25 \mathrm{mM} \text { versus } \\
1.75 \mathrm{mM} \\
\text { (observational study } \\
\text { of prevalent cases) }\end{array}$ & 6 months & Brachial PWV, augmentation index (AI) & $\begin{array}{l}\text { (i) PWV identical at } \\
\text { baseline in both groups, } \\
\text { (ii) PWV increased in the } \\
\text { high-calcium group during } \\
\text { study (not adjusted for BP) }\end{array}$ \\
\hline
\end{tabular}

HD: hemodialysis; PD: peritoneal dialysis; BP: blood pressure; iCa: ionized calcium; $\triangle \mathrm{iCa}$ : changes in iCa; PWV: pulse wave velocity; AC: arterial compliance; AI: augmentation index.

to its baseline value of $0.140 \mathrm{~mm}^{2} / \mathrm{kPa}$. In this study, the iCa was $1.6 \pm 0.1 \mathrm{mM}$ at baseline, decreased to $1.11 \pm 0.07 \mathrm{mM}$ with DCa of $1.25 \mathrm{mM}$ ( $P<.05$ compared to baseline), and returned to baseline levels $(1.6 \pm 0.1)$ after reusing DCa of $1.75 \mathrm{mM}$.

In another study by Kyriazis and colleagues [26], the effects of DCa on arterial stiffness were studied during a single session of dialysis with DCa of 1.75 versus $1.25 \mathrm{mM}$. In this study arterial stiffness was assessed by stiffness index (SI) and reflection index (RI) that were derived from pulse wave analysis of digital volume pulse (DVP). The DVP waveform consists of a systolic peak and a second diastolic peak which is formed by the reflection of the pulse wave from the reflection site from the lower body (Figure 5). The time delay (peakto-peak time (PPT), see Figure 5) between the systolic and diastolic peaks is related to the transit time of pressure waves from the root of the subclavian artery to the apparent site of reflection and back to the subclavian artery. In addition to conduit vessel stiffness, the degree of pulse wave reflection also depends on the impedance of the microvascular bed and the tone of the small- to medium-sized blood vessels. Knowing that the reflection site is proportional to the height (h) of the subject, the stiffness index is calculated by dividing the height by the peak-to-peak time (SI = h/PPT) [27]. The $\mathrm{RI}$ is calculated by dividing the height of the reflective wave (b) to the height of the incident wave (a) (RI = b/a) [27]. Therefore, SI is determined both by pulse wave velocity and vascular tone, while RI is a measure of pulse wave reflection. [28] In this protocol, both SI and RI increased, respectively, by 5.7 and $6 \%$ during treatment with a DCa of $1.75 \mathrm{mM}$, whereas they remained unchanged with DCa of $1.25 \mathrm{mM}$. Serum iCa increased with $1.75 \mathrm{mM}(1.15 \pm 0.08$ versus 1.65 $\pm 0.07, P<.001)$ and decreased with $1.25 \mathrm{mM}(1.16 \pm 0.09$ versus $1.10 \pm 0.05, P<.001)$.

The historical choice of a DCa of 1.75 and even $1.5 \mathrm{mM}$ in an era where calcium-based phosphate binders were universally used in CKD patients was gradually challenged over the last decade. In this context, we undertook the task of evaluating the impact of three DCa $(1.00,1.25,1.5 \mathrm{mM})$ that changed the postdialysis ionized calcium concentrations within the physiological range [29]. Accordingly, 18 subjects underwent a midweek dialysis session with all three DCa over a three-week period. Arterial stiffness was assessed before and after each dialysis. Carotid-femoral PWV (c-f PWV), and carotid-radial PWV (c-r PWV) was measured to determine the stiffness of both elastic and muscular conduit arteries. The central hemodynamic impact of DCa was determined by means of generalized transfer function applied to radial pulse wave profile. In postdialysis, iCa decreased with DCa of $1.00 \mathrm{mM}(-0.14 \pm 0.04 \mathrm{mM}, P<.001)$, increased with 


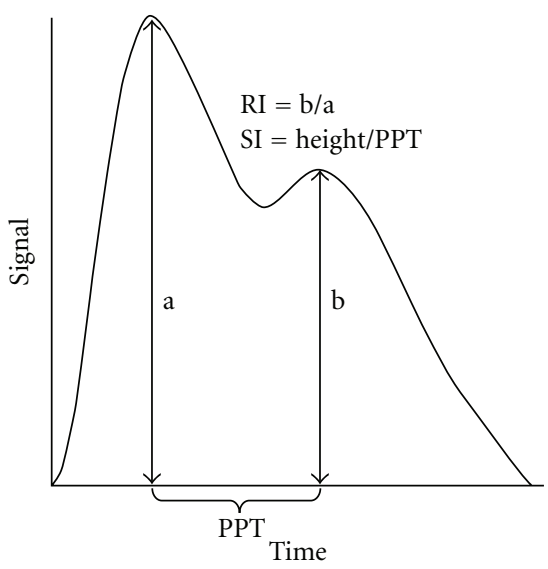

Figure 5: Contour of digital volume pulse. The digital volume pulse provides a dicrotic signal. The first peak is the incident pulse wave generated from the heart while the second peak is generated from the reflection of incidental wave from the reflection site of the lower part of the body. The peak-to-peak time (PPT) represents the transit time between the incidental and reflective waves. Since the travel distance of the reflective wave is proportional to the height (h) of the subject, a stiffness index (SI) can be calculated by dividing height by the PPT. The ratio of the height of the reflective wave (b) to the incidental wave (a) is used as the reflective index (RI).

a DCa of $1.50 \mathrm{mM}(0.10 \pm 0.06 \mathrm{mM}, P<.001)$ but did not change with a DCa of $1.25 \mathrm{mM}$. Tests of withinsubject contrast showed a linear relationship between higher DCa and higher postdialysis changes in c-f PWV, c-r PWV, and mean blood pressure $(P<.001, P=.008$ and $P=.002$, resp.). Heart rate-adjusted central augmentation index (augmentation pressure divided pulse pressure, see Figure 4) decreased significantly after dialysis, but was not related to DCa. In a multivariate linear-mixed model for repeated measures, the percentage increase in c-f PWV and c-r PWV was significantly associated with the increasing level of $\mathrm{iCa}$, whereas the increasing level of change in mean blood pressure was not significant. We conclude that DCa and acute changes in the serum iCa concentration, even within physiological range, are associated with detectable and significant changes of arterial stiffness.

\section{Impact of Dialysis Calcium Concentration on Arterial Stiffness in Peritoneal Dialysis}

The impact of peritoneal DCa on arterial stiffness still remains elusive. The only data available comes from a recent report from Demirci et al. [30] where they evaluated the effects of DCa on the progression of arterial stiffness. These patients were on dialysis for more than 6 months with either DCa of $1.25(n=34)$ or $1.75 \mathrm{mM}(n=15)$. After a baseline assessment of arterial stiffness, the patients were reevaluated 6 months later while using the same dialysis prescription. Arterial stiffness was assessed by brachial artery PWV, and central augmentation index was determined by radial artery tonometry. At baseline, the augmentation index was higher with DCa $1.75 \mathrm{mM}$ as compared to $1.25 \mathrm{mM}$
$(27 \% \pm 10 \%$ versus $21 \% \pm 9 \%, P<.05)$. Brachial PWV was not different between the groups at baseline $(8.4 \pm 1.1 \mathrm{~m} / \mathrm{s}$ versus $8.5 \pm 1.7 \mathrm{~m} / \mathrm{s}, \mathrm{p}=\mathrm{ns})$. However, after 6 months, brachial PWV increased in the $1.75 \mathrm{mM}$ group (from $8.4 \pm$ 1.1 to $9.6 \pm 2.3 \mathrm{~m} / \mathrm{s}, P<.05)$, but had not changed in the $1.25 \mathrm{mM}$ group (from $8.5 \pm 1.7 \mathrm{~m} / \mathrm{s}$ to $8.2 \pm 1.9 \mathrm{~m} / \mathrm{s}, P=\mathrm{ns}$ ). The augmentation index did not progress in either group. There are however some limitations to the interpretation of the results provided by this study. First, the blood pressure was higher in the $1.75 \mathrm{mM}$ group as compared to the $1.25 \mathrm{mM}$ group at baseline $(100 \pm 22 \mathrm{mmHg}$ versus $88 \pm$ $18 \mathrm{mmHg}, P=.06)$ and still higher after 6 months of followup (106 \pm 14 versus $91 \pm 15, P<.01$ ( $P$ value was not provided by the original authors)). Therefore, it cannot be assumed that the progression of arterial stiffness was blood pressure independent in this study. Second, it should be mentioned that these subjects were at least on the same DCa for the preceding six months prior to baseline evaluation, and yet their brachial PWV was practically identical at baseline. Therefore, it is hard to reconcile why an impressive progression of brachial artery stiffness occurred over the ensuing 6 months in the $1.75 \mathrm{mM}$ group.

\section{Conclusion}

In summary, it is clear that acute manipulations of serum ionized calcium by dialysis calcium concentration can modulate vascular stiffness. This behavior can readily be explained on the basis of smooth muscle cells being in series with collagen and in parallel with elastin fibers, such that reduction in muscular tone transfers stress from the muscular fibers and collagen to the elastins of the wall, and the increase in muscular tone produces the reverse effect $[31,32]$. However, in long-term studies, the DCa could also lead to structural arterial stiffness that may result from increased calcium load and its interference with mineral and bone metabolism. Clearly, long-term studies are required to evaluate the impact of DCa on the progression of arterial stiffness to better define the optimal dialysis calcium concentration.

\section{References}

[1] J. Blacher, A. P. Guerin, B. Pannier, S. J. Marchais, M. E. Safar, and G. M. London, "Impact of aortic stiffness on survival in end-stage renal disease," Circulation, vol. 99, no. 18, pp. 24342439, 1999.

[2] J. Blacher, B. Pannier, A. P. Guerin, S. J. Marchais, M. E. Safar, and G. M. London, "Carotid arterial stiffness as a predictor of cardiovascular and all- cause mortality in end-stage renal disease," Hypertension, vol. 32, no. 3, pp. 570-574, 1998.

[3] P. Boutouyrie, A. I. Tropeano, R. Asmar et al., "Aortic stiffness is an independent predictor of primary coronary events in hypertensive patients: a longitudinal study," Hypertension, vol. 39, no. 1, pp. 10-15, 2002.

[4] S. Laurent, P. Boutouyrie, R. Asmar et al., "Aortic stiffness is an independent predictor of all-cause and cardiovascular mortality in hypertensive patients," Hypertension, vol. 37, no. 5, pp. 1236-1241, 2001. 
[5] C. Vlachopoulos, K. Aznaouridis, and C. Stefanadis, "Prediction of cardiovascular events and all-cause mortality with arterial stiffness: a systematic review and meta-analysis," Journal of the American College of Cardiology, vol. 55, no. 13, pp. 1318-1327, 2010.

[6] J. Apter and M. Rabinowitz, "Correlation of visco-elastic properties of large arteries with microscopic structure. (1) Methods used and their justification. (2) Elastin and muscle determined chemically," Circulation Research, vol. 19, pp. 104121, 1966.

[7] M. F. O’Rourke, "Pulsatile arterial haemodynamics in hypertension," Australian and New Zealand Journal of Medicine, vol. 6, supplement 2, pp. 40-48, 1976.

[8] B. H. R. Wolffenbuttel, C. M. Boulanger, F. R. L. Crijns et al., "Breakers of advanced glycation end products restore large artery properties in experimental diabetes," Proceedings of the National Academy of Sciences of the United States of America, vol. 95, no. 8, pp. 4630-4634, 1998.

[9] H. H. Dao, R. Essalihi, C. Bouvet, and P. Moreau, "Evolution and modulation of age-related medial elastocalcinosis: impact on large artery stiffness and isolated systolic hypertension," Cardiovascular Research, vol. 66, no. 2, pp. 307-317, 2005.

[10] S. E. Greenwald, "Ageing of the conduit arteries," Journal of Pathology, vol. 211, no. 2, pp. 157-172, 2007.

[11] M. L. Harkness, R. D. Harkness, and D. A. Mcdonald, "The collagen and elastin content of the arterial wall in the dog," Proceedings of the Royal Society B: Biological Sciences, vol. 146, no. 925, pp. 541-551, 1957.

[12] B. Pannier, A. P. Guérin, S. J. Marchais, M. E. Safar, and G. M. London, "Stiffness of capacitive and conduit arteries: prognostic significance for end-stage renal disease patients," Hypertension, vol. 45, no. 4, pp. 592-596, 2005.

[13] S. Laurent, S. Katsahian, C. Fassot et al., "Aortic stiffness is an independent predictor of fatal stroke in essential hypertension," Stroke, vol. 34, no. 5, pp. 1203-1206, 2003.

[14] G. Mulè, S. Cottone, P. Cusimano et al., "The association of microalbuminuria with aortic stiffness is independent of creactive protein in essential hypertension," American Journal of Hypertension, vol. 22, no. 10, pp. 1041-1047, 2009.

[15] G. Mulè, S. Cottone, A. Vadalà et al., "Relationship between albumin excretion rate and aortic stiffness in untreated essential hypertensive patients," Journal of Internal Medicine, vol. 256, no. 1, pp. 22-29, 2004.

[16] A. Upadhyay, S. J. Hwang, G. F. Mitchell et al., "Arterial stiffness in mild-to-moderate CKD," Journal of the American Society of Nephrology, vol. 20, no. 9, pp. 2044-2053, 2009.

[17] A. Adji, M. F. O'Rourke, and M. Namasivayam, "Arterial stiffness, its assessment, prognostic value, and implications for treatment," American Journal of Hypertension, vol. 24, no. 1, pp. 5-17, 2011.

[18] S. Laurent, J. Cockcroft, L. Van Bortel et al., "Expert consensus document on arterial stiffness: methodological issues and clinical applications," European Heart Journal, vol. 27, no. 21, pp. 2588-2605, 2006.

[19] W. L. Henrich, J. M. Hunt, and J. V. Nixon, "Increased ionized calcium and left ventricular contractility during hemodialysis," New England Journal of Medicine, vol. 310, no. 1, pp. 19-23, 1984.

[20] W. H. M. Van Kuijk, A. W. Mulder, C. H. Peels, G. A. Harff, and K. M. L. Leunissen, "Influence of changes in ionized calcium on cardiovascular reactivity during hemodialysis," Clinical Nephrology, vol. 47, no. 3, pp. 190-196, 1997.
[21] F. M. Van Der Sande, E. C. Cheriex, W. H. M. Van Kuijk, and K. M. L. Leunissen, "Effect of dialysate calcium concentrations on intradialytic blood pressure course in cardiac-compromised patients," American Journal of Kidney Diseases, vol. 32, no. 1, pp. 125-131, 1998.

[22] L. Gabutti, G. Bianchi, D. Soldini, C. Marone, and M. Burnier, "Haemodynamic consequences of changing bicarbonate and calcium concentrations in haemodialysis fluids," Nephrology Dialysis Transplantation, vol. 24, no. 3, pp. 973-981, 2009.

[23] S. Marchais, A. Guerin, M. Safar, and G. London, "Arterial compliance in uraemia," Journal of Hypertension. Supplement, vol. 7, no. 6, pp. S84-S85, 1989.

[24] J. Kyriazis, D. Stamatiadis, and A. Mamouna, "Intradialytic and interdialytic effects of treatment with 1.25 and $1.75 \mathrm{mmol} / \mathrm{L}$ of calcium dialysate on arterial compliance in patients on hemodialysis," American Journal of Kidney Diseases, vol. 35, no. 6, pp. 1096-1103, 2000.

[25] S. J. Yoo, D. J. Oh, and S. H. Yu, "The effects of low calcium dialysate on arterial compliance and vasoactive substances in patients with hemodialysis," Korean Journal of Internal Medicine, vol. 19, no. 1, pp. 27-32, 2004.

[26] J. Kyriazis, I. Katsipi, K. Stylianou, N. Jenakis, A. Karida, and E. Daphnis, "Arterial stiffness alterations during hemodialysis: the role of dialysate calcium," Nephron-Clinical Practice, vol. 106, no. 1, pp. c34-c42, 2007.

[27] S. C. Millasseau, J. M. Ritter, K. Takazawa, and P. J. Chowienczyk, "Contour analysis of the photoplethysmographic pulse measured at the finger," Journal of Hypertension, vol. 24 , no. 8, pp. 1449-1456, 2006.

[28] D. Sollinger, M. G. Mohaupt, A. Wilhelm, D. Uehlinger, F. J. Frey, and U. Eisenberger, "Arterial stiffness assessed by digital volume pulse correlates with comorbidity in patients with ESRD," American Journal of Kidney Diseases, vol. 48, no. 3, pp. 456-463, 2006.

[29] A. Lebeouf, F. Mac-Way, M. S. Utescu et al., "Effects of acute variation of dialysate calcium concentrations on arterial stiffness and aortic pressure waveform," Nephrology Dialysis Transplantation, vol. 24, no. 12, pp. 3788-3794, 2009.

[30] M. S. Demirci, M. Ozkahya, G. Asci et al., "The influence of dialysate calcium on progression of arterial stiffness in peritoneal dialysis patients," Peritoneal Dialysis International, vol. 29, supplement 2, pp. S15-S17, 2009.

[31] A. Rachev and K. Hayashi, "Theoretical study of the effects of vascular smooth muscle contraction on strain and stress distributions in arteries," Annals of Biomedical Engineering, vol. 27, no. 4, pp. 459-468, 1999.

[32] W. Nichols, M. O'Rourke, and M. McDonald's, Blood Flow in Arteries, Hodder Arnold, London, UK, 5th edition, 2005. 


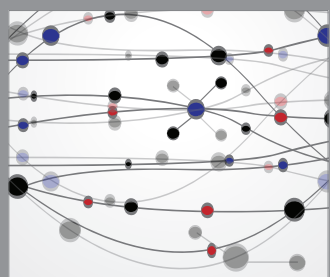

The Scientific World Journal
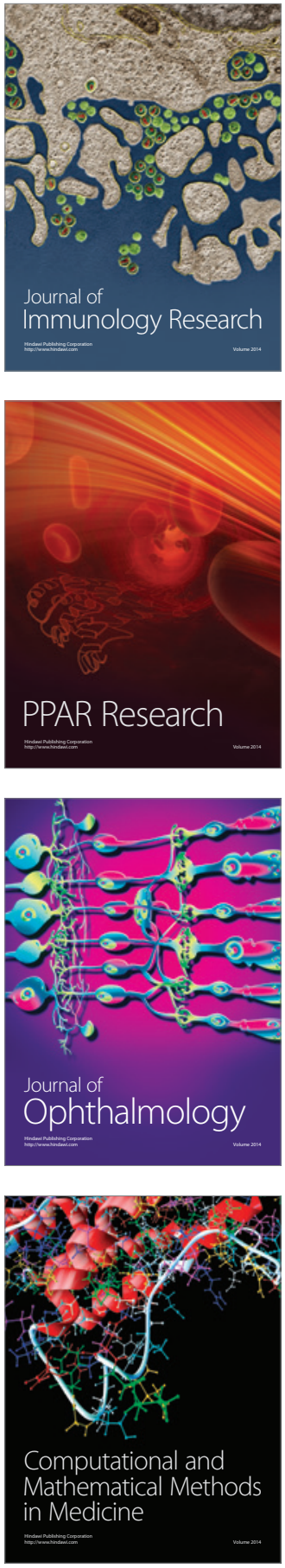

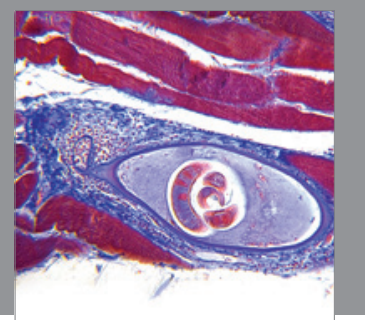

Gastroenterology

Research and Practice
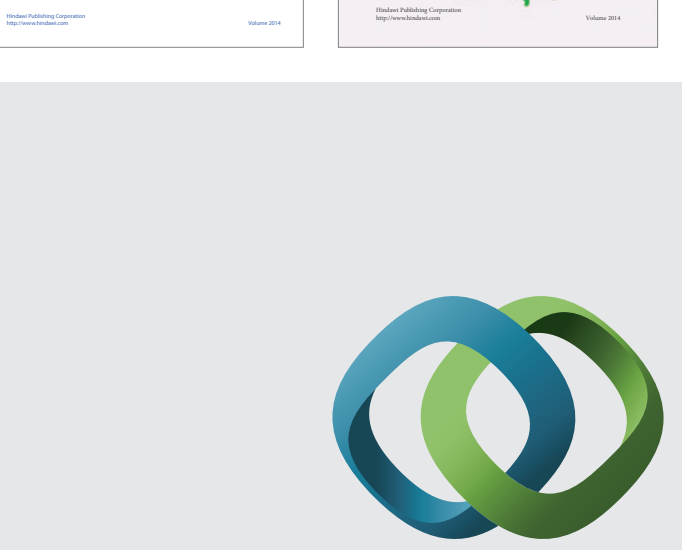

\section{Hindawi}

Submit your manuscripts at

http://www.hindawi.com
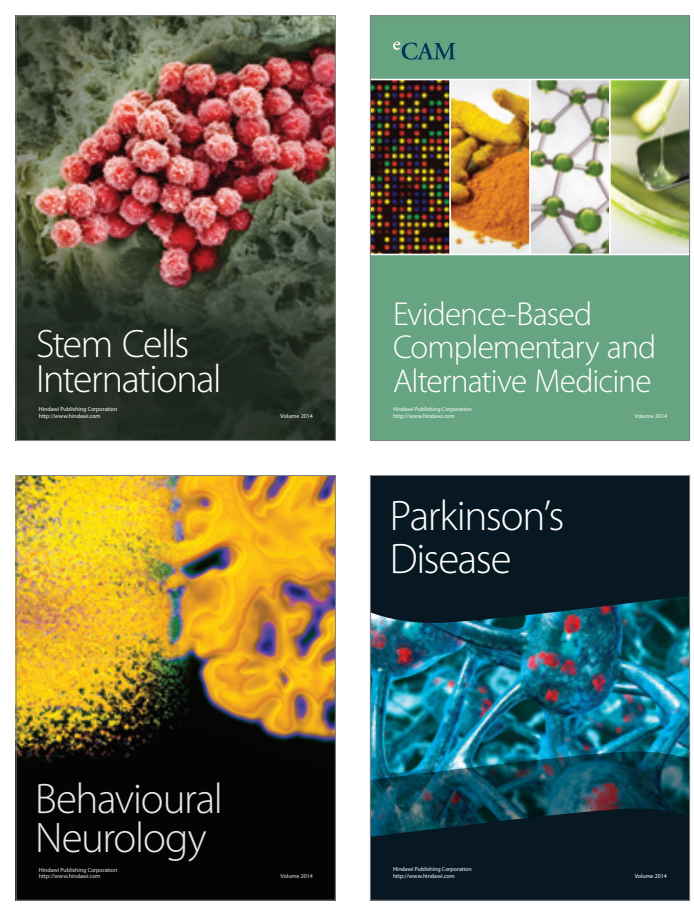

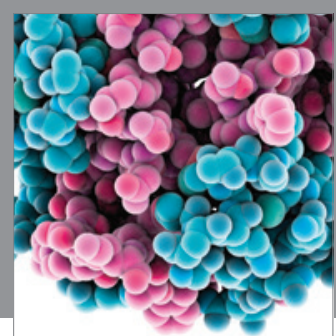

Journal of
Diabetes Research

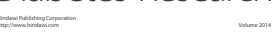

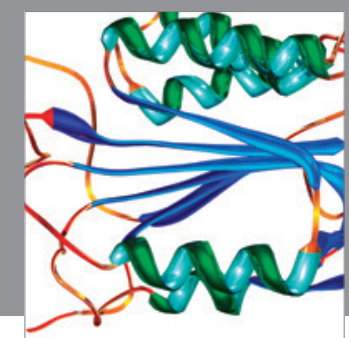

Disease Markers
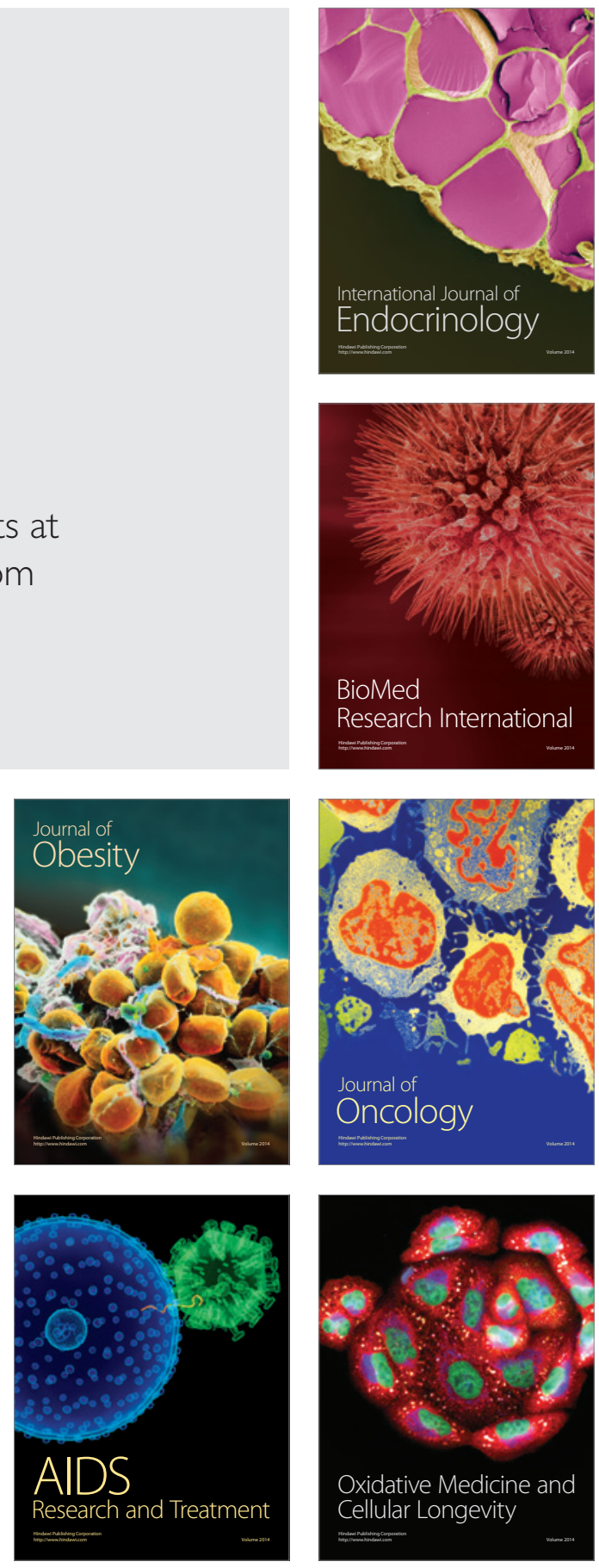